\title{
Low-frequency internal waves in magnetized rotating stellar radiation zones
}

\section{Wave structure modification by a toroidal field}

\author{
S. Mathis ${ }^{1,2}$ and N. de Brye ${ }^{1}$ \\ ${ }^{1}$ Laboratoire AIM, CEA/DSM - CNRS - Université Paris Diderot, IRFU/SAp Centre de Saclay, 91191 Gif-sur-Yvette, France \\ e-mail: stephane.mathis@cea.fr, nicolas.de-brye@obspm.fr \\ 2 LESIA, Observatoire de Paris - CNRS - Université Paris Diderot, Place Jules Janssen, 92195 Meudon, France
}

Received 11 August 2010 / Accepted 23 September 2010

\begin{abstract}
Context. The study of helioseismology, asteroseismology, and powerful ground-based instrumentation dedicated to stellar physics is developing strongly (cf. CoRoT, KEPLER, and ESPaDOnS). This generates tight constraints on the stellar internal structure and dynamical processes. In this context, it is thus necessary to go beyond the non-rotating and the non-magnetic picture of stellar interiors, particularly for large-scale transport mechanisms and waves.

Aims. We focus on low-frequency internal waves in magnetic, rotating, stably stratified stellar radiation zones. For frequencies, which can be close to the Alfvén and the inertial frequencies, we go beyond the non-magnetic and non-rotating description of wave dynamics with taking the Coriolis acceleration and the Lorentz force into account. Then, we have to couple wave dynamics with fossil magnetic fields, which must have mixed configurations (both poloidal and toroidal) to survive in stellar radiation zones.

Methods. We chose to study such coupling step by step, first with purely toroidal fields and then with purely poloidal fields, to unravel their modification by each corresponding component of a realistic mixed-field. Thus, we analytically built a complete formalism, which describes both effects of the Coriolis acceleration and of the Lorentz force in a non-perturbative way in the case of an axisymmetric toroidal field. We consider here the case where both Alfvén frequency and angular velocity are chosen to be uniform, to isolate wave properties.

Results. The different approximations possible for low-frequency internal waves in this model are examined and discussed. In this way, the traditional approximation used to describe the dynamics of low-frequency regular elliptic gravito-inertial waves in the purely hydrodynamical case is generalized to the magnetic one. The complete structure of internal waves, which become magneto-gravitoinertial waves, is then derived and compared to the non-magnetic case. The asymptotic behaviour of such waves is obtained.

Conclusions. A global study of magneto-gravito-inertial waves in stellar radiation zones is achieved in the case of an axisymmetric toroidal magnetic field. In the near future, consequences for angular momentum transport and the case of general differential rotation and azimuthal magnetic field have to be studied. Moreover, the same methodology must be applied to the case of poloidal fields, and the hyperbolic regime has to be carefully studied.
\end{abstract}

Key words. magnetohydrodynamics (MHD) - waves - methods: analytical - stars: rotation - stars: magnetic field stars: oscillations

\section{Introduction}

Stellar radiation zones are stable strongly stratified rotating magnetic regions. This means that fluid dynamics in such zones are driven by the buoyancy force, the Coriolis and the centrifugal accelerations, and the Lorentz force. Furthermore, those regions are the seat of transport and mixing processes that rule the secular evolution of stars along with nuclear reactions (see for example Zahn 1992; Meynet \& Maeder 2000). Then, angular momentum, heat, magnetic field, and chemicals are transported, leading to a modification of the stellar structure.

Observations from space and ground (with helioseismology, asteroseismology, and high-resolution spectropolarimetry, for example) now give us ever more refined constraints on physical processes in star interiors. In this way, we have to built models that become more and more realistic, now taking internal dynamical processes into account. A complete review of those mechanisms in stellar radiation zones is given by Talon (2008).
In this work, we focus on the waves that propagate in such regions, namely the internal (or gravity) waves. These waves transport angular momentum along with fossil magnetic field (Mestel et al. 1988; Gough \& McIntyre 1998; Brun \& Zahn 2006; Garaud \& Guervilly 2009) and are serious candidates for explaining stellar radiation zone angular velocity profiles, as in the Sun and solar-type stars (cf. Talon et al. 2002; Talon \& Charbonnel 2005; Charbonnel \& Talon 2005; Denissenkov et al. 2008). Since stellar radiation regions are differentially rotating and magnetic (for example, in the solar tachocline where waves are excited; see Browning et al. 2006), internal waves dynamics are modified by the Coriolis acceleration (the centrifugal one can be neglected to the first order in rotation) and by the Lorentz force, thus becoming magneto-gravito-inertial waves (Schatzman 1993a; Barnes et al. 1998; Kumar et al. 1999; Schecter et al. 2001; Rogers \& MacGregor 2010). They are equivalent to the MAC waves (for magnetic-ArchimedeanCoriolis) studied in geophysics for the liquid Earth's core (see 
Braginsky 1967; Braginsky \& Roberts 1975; Friedlander 1987b, 1989), but the relative importance of each force is however different in the stellar case with a strong domination of the stratification restoring force. For the fossil magnetic field configuration in stellar radiation zones, the simplest geometrical configurations, such as purely poloidal and purely toroidal fields, are known to be unstable (Tayler 1973; Achesson 1978; Markey \& Tayler 1973, 1974), with the best candidate for stable geometries the mixed poloidal-toroidal fields (Wright 1973; Markey \& Tayler 1974; Tayler 1980; Braithwaite \& Spruit 2004; Braithwaite 2009; Duez \& Mathis 2010). However, it is a hard and very new task to consider such a complex helical field geometry in the study of internal wave dynamics. This is the reason we chose to treat the modification of wave propagation, and the transport they induce, first by a purely toroidal field, and second by a purely poloidal field (see for example Lee 2005-2007-2010 in the dipolar case), to unravel their modification by each corresponding component of a realistic mixed field.

The wave dynamics equations are given here for an axisymmetric toroidal (and thus azimuthal) magnetic field in Sect. 2. Examining a model of rotating and magnetic stellar radiation zone where the angular velocity and the Alfvén frequency are assumed to be uniform (cf. Fig. 1), which constitutes the first step in understanding the complex behaviour of such waves, we introduce in Sect. 3 the so-called traditional approximation, which we generalize to the MHD case. In this framework, we derive the complete spatial structure of magneto-gravito-inertial waves and their asymptotic properties. Then, the validity of the MHD traditional approximation is discussed in Sect. 4 and wave families are identified. Finally, conclusions are given and perspectives for modifying wave-induced transport of angular momentum are discussed in Sect. 5.

\section{Waves in rotating stars with a toroidal magnetic field}

To study the dynamics of magneto-gravito-inertial waves in stellar radiation zones, the classical perfect MHD inviscid dynamical equations system has to be solved. It is formed by the induction equation in the non-dissipative case (here, the ohmic dissipation is not taken into account because it is negligible compared to the thermal diffusion)

$\partial_{t} \boldsymbol{B}=\boldsymbol{\nabla} \times(\boldsymbol{V} \times \boldsymbol{B})$,

the momentum equation

$D_{t} \boldsymbol{V}=-\frac{1}{\rho} \nabla P-\nabla \Phi+\frac{1}{\rho}\left[\frac{1}{\mu}(\boldsymbol{\nabla} \times \boldsymbol{B}) \times \boldsymbol{B}\right]$,

the continuity equation

$D_{t} \rho+\rho \boldsymbol{\nabla} \cdot \boldsymbol{V}=0$,

the equation for the energy transport, which is given here in the adiabatic limit

$\frac{1}{\Gamma_{1}} D_{t} \ln P-D_{t} \ln \rho=0$

and the Poisson's equation for the gravitational potential

$\nabla^{2} \Phi=4 \pi G \rho$.

Here, $\boldsymbol{B}$ is the macroscopic magnetic field. It is formed by the sum of the large-scale toroidal field $\boldsymbol{B}_{0}^{\mathrm{T}}$, associated to the chosen

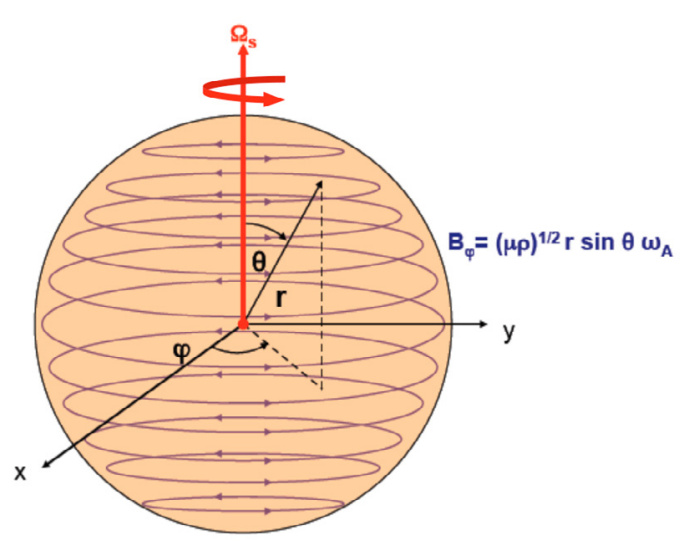

Fig. 1. The set-up to study internal wave dynamics in rotating and magnetic stellar radiation zones. Angular velocity $\left(\Omega_{\mathrm{s}}\right)$ and Alfvén frequency $\left(\omega_{\mathrm{A}}\right)$ are assumed uniform in a first step to unravel magnetogravito-inertial wave characteristics.

uniform Alfvén frequency $\omega_{\mathrm{A}}{ }^{1}$, and of the wave-induced magnetic field $\boldsymbol{b}$ :

$\boldsymbol{B}(\boldsymbol{r}, t)=\boldsymbol{B}_{0}^{\mathrm{T}}(\boldsymbol{r}, t)+\boldsymbol{b}(\boldsymbol{r}, t)$ with $\boldsymbol{B}_{0}^{\mathrm{T}}=\sqrt{\mu \rho} r \sin \theta \omega_{\mathrm{A}} \widehat{\boldsymbol{e}}_{\varphi}$,

where $t$ is the time and $\boldsymbol{r}=(r, \theta, \varphi)$ are the usual spherical coordinates with their associated unit vector basis $\left\{\widehat{\boldsymbol{e}}_{k}\right\}_{k=\{r, \theta, \varphi\}}, \rho$ is the density, and $\mu$ is the magnetic permeability of the medium. Next, $\boldsymbol{V}$ is the macroscopic velocity field. It is formed by the sum of the large-scale azimuthal velocity $\boldsymbol{V}_{0}$, associated to the chosen uniform rotation $\Omega_{\mathrm{s}}$, and of the wave velocity $\boldsymbol{u}$ :

$\boldsymbol{V}(\boldsymbol{r}, t)=\boldsymbol{V}_{0}(\boldsymbol{r}, t)+\boldsymbol{u}(\boldsymbol{r}, t)$ with $\boldsymbol{V}_{0}=r \sin \theta \Omega_{\mathrm{s}} \widehat{\boldsymbol{e}}_{\varphi}$,

where $D_{t}=\partial_{t}+(\boldsymbol{V} \cdot \boldsymbol{\nabla})$ is the Lagrangian derivative. The $\Phi$ and $P$ are the gravitational potential and the pressure, while $\Gamma_{1}=(\partial \ln P / \partial \ln \rho)_{S}$ is the adiabatic exponent, with $S$ the macroscopic entropy, and $G$ the universal gravitational constant.

To achieve our aim, Eqs. (1)-(5) are linearized around the rotating magnetic steady state. Each scalar field $X$ (the density, the gravitational potential, and the pressure) is then expanded as the sum of its hydrostatic value $\bar{X}$ and of the wave's associated fluctuation $\widetilde{X}$ :

$X(r, \theta, \varphi, t)=\bar{X}(r)+\widetilde{X}(r, \theta, \varphi, t)$.

We neglect the non-spherical character of the hydrostatic background due to the deformation associated to the centrifugal acceleration, $\gamma_{\mathrm{c}}\left(\Omega_{\mathrm{S}}\right)=1 / 2\left[\Omega_{\mathrm{s}}^{2} \nabla\left(r^{2} \sin ^{2} \theta\right)\right]$, and to the Lorentz force associated to $\boldsymbol{B}_{0}^{\mathrm{T}}, \boldsymbol{F}_{\mathcal{L}}^{0}\left(\boldsymbol{B}_{0}^{\mathrm{T}}\right)=1 / \mu\left[\left(\boldsymbol{\nabla} \times \boldsymbol{B}_{0}^{\mathrm{T}}\right) \times \boldsymbol{B}_{0}^{\mathrm{T}}\right]$.

Then, following Braginsky (1967), Braginsky \& Roberts (1975), and Unno et al. (1989), the wave's Lagrangian displacement $\boldsymbol{\xi}$ is introduced and defined such that

$\boldsymbol{u}=\partial_{t} \boldsymbol{\xi}+\left(\boldsymbol{V}_{0} \cdot \boldsymbol{\nabla}\right) \boldsymbol{\xi}-(\boldsymbol{\xi} \cdot \boldsymbol{\nabla}) \boldsymbol{V}_{0}$.

1 The Alfvén speed associated to an axisymmetric toroidal magnetic field $\left(B_{0}^{\mathrm{T}}\right)$ is given by $V_{\mathrm{A}}(r, \theta)=B_{0}^{\mathrm{T}}(r, \theta) / \sqrt{\mu \rho}$. Since $\omega_{\mathrm{A}}=V_{\mathrm{A}} / s$, where $s=r \sin \theta$ is the distance from the symmetry axis, we get $B_{0}^{\mathrm{T}}(r, \theta)=\sqrt{\mu \rho} r \sin \theta \omega_{\mathrm{A}}(r, \theta)$. Here, we choose a uniform $\omega_{\mathrm{A}}$ as a first step. The case of a general realistic axisymmetric toroidal field $\left(\omega_{\mathrm{A}}(r, \theta)\right)$ will be treated in Paper III. 
S. Mathis and N. de Brye: Internal wave structure in magnetized rotating stellar radiation zones. I.

Using Eq. (7), it becomes

$\boldsymbol{u}=\left(\partial_{t}+\Omega_{\mathrm{s}} \partial_{\varphi}\right) \boldsymbol{\xi}$

Next, inserting Eq. (9) in the induction equation (Eq. (1)), we get

$\boldsymbol{b}=\boldsymbol{\nabla} \times\left(\boldsymbol{\xi} \times \boldsymbol{B}_{0}^{\mathrm{T}}\right)$.

Using the definition of $\boldsymbol{B}_{0}^{\mathrm{T}}$ given in Eq. (6), this leads to

$\boldsymbol{b}=\sqrt{\mu \bar{\rho}} \omega_{\mathrm{A}} \partial_{\varphi} \boldsymbol{\xi}$.

Then, the momentum equation (Eq. (2)) becomes

$\left(\partial_{t}+\Omega_{\mathrm{s}} \partial_{\varphi}\right)\left[\left(\partial_{t}+\Omega_{\mathrm{s}} \partial_{\varphi}\right) \boldsymbol{\xi}+2 \Omega_{\mathrm{s}} \widehat{\boldsymbol{e}}_{z} \times \boldsymbol{\xi}\right]=$

$-\frac{1}{\bar{\rho}} \boldsymbol{\nabla} \Pi(\boldsymbol{r}, t)-\boldsymbol{\nabla} \widetilde{\Phi}+\frac{\widetilde{\rho}}{\bar{\rho}^{2}} \boldsymbol{\nabla} \bar{P}+\frac{\boldsymbol{F}_{\mathcal{L}}^{\mathrm{Te}}(\boldsymbol{\xi})}{\bar{\rho}}$,

where $\widehat{\boldsymbol{e}}_{z}=\cos \theta \widehat{\boldsymbol{e}}_{r}-\sin \theta \widehat{\boldsymbol{e}}_{\theta}$ is the unit vector along the rotation axis. Next, the wave total pressure fluctuation is given by the sum of the gas pressure fluctuation $\widetilde{P}$ and of the wave magnetic pressure $\widetilde{P}_{\mathrm{M}}(\boldsymbol{r}, t)=\left(\boldsymbol{B}_{0}^{\mathrm{T}} \cdot \boldsymbol{b}\right) / \mu$ :

$\Pi=\widetilde{P}+\frac{\boldsymbol{B}_{0}^{\mathrm{T}} \cdot \boldsymbol{b}}{\mu}$.

Afterwards, the wave magnetic tension force is given by

$$
\begin{aligned}
\boldsymbol{F}_{\mathcal{L}}^{\mathrm{Te}}(\boldsymbol{\xi}) & =\frac{1}{\mu}\left[\left(\boldsymbol{B}_{0}^{\mathrm{T}} \cdot \boldsymbol{\nabla}\right) \boldsymbol{b}+(\boldsymbol{b} \cdot \boldsymbol{\nabla}) \boldsymbol{B}_{0}^{\mathrm{T}}\right] \\
& =\bar{\rho} \omega_{\mathrm{A}}^{2}\left[\partial_{\varphi^{2}} \boldsymbol{\xi}+2 \widehat{\boldsymbol{e}}_{z} \times \partial_{\varphi} \boldsymbol{\xi}\right] .
\end{aligned}
$$

Finally, the continuity (Eq. (3)), the energy transport (Eq. (4)), and the Poisson's (Eq. (5)) equations are respectively given by

$\widetilde{\rho}+\boldsymbol{\nabla} \cdot(\bar{\rho} \boldsymbol{\xi})=0$,

$\left(\frac{\widetilde{P}}{\Gamma_{1} \bar{P}}-\frac{\widetilde{\rho}}{\bar{\rho}}\right)+\xi \cdot\left(\frac{1}{\Gamma_{1}} \nabla \ln \bar{P}-\nabla \ln \bar{\rho}\right)=0$,

$\nabla^{2} \widetilde{\Phi}=4 \pi G \widetilde{\rho}$.

Next, $\widetilde{X}$ and each vectorial field $\boldsymbol{x}(\boldsymbol{r}, t)=\{\boldsymbol{u}, \boldsymbol{\xi}, \boldsymbol{b}\}$ are expanded in Fourier's series in $\varphi$ and $t$ :

$\widetilde{X}=\sum_{\sigma, m}\left\{X^{\prime}(r, \theta) \exp (\mathrm{i} m \varphi) \exp (\mathrm{i} \sigma t)\right\}$

$\boldsymbol{x}=\sum_{\sigma, m}\left\{\boldsymbol{x}^{\prime}(r, \theta) \exp (\mathrm{i} m \varphi) \exp (\mathrm{i} \sigma t)\right\}$

with $\sigma$ the wave angular velocities in an inertial frame excited in the star.

On one hand, we get for the velocity field

$\boldsymbol{u}^{\prime}=\mathrm{i} \sigma_{\mathrm{s}} \boldsymbol{\xi}^{\prime}$ with $\sigma_{\mathrm{s}}=\sigma+m \Omega_{\mathrm{s}}$.

In the considered rotating stellar radiation zone, waves are thus Doppler-shifted by rotation $\left(\Omega_{\mathrm{s}}\right)$ and the local wave angular velocity $\left(\sigma_{\mathrm{s}}\right)$ that corresponds to the operator $\partial_{t}+\Omega_{\mathrm{s}} \partial_{\varphi}$ appears.

On the other hand, Eq. (12) becomes

$\boldsymbol{b}^{\prime}=\mathrm{i} m \sqrt{\mu \bar{\rho}} \omega_{\mathrm{A}} \boldsymbol{\xi}^{\prime}$
Then, the momentum equation is written as

$$
-\mathcal{A} \xi^{\prime}+\mathrm{i} \mathcal{B} \widehat{\boldsymbol{e}}_{z} \times \xi^{\prime}=-\nabla W^{\prime}+\frac{\rho^{\prime}}{\bar{\rho}^{2}} \nabla \bar{P}-\Pi^{\prime} \frac{\nabla \bar{\rho}}{\bar{\rho}^{2}},
$$

where

$\mathcal{A}=\sigma_{\mathrm{M}}^{2}=\sigma_{\mathrm{s}}^{2}-m^{2} \omega_{\mathrm{A}}^{2} \quad$ and $\quad \mathcal{B}=2\left(\Omega_{\mathrm{s}} \sigma_{\mathrm{s}}-m \omega_{\mathrm{A}}^{2}\right)$.

Expanding each component explicitely gives along $\widehat{\boldsymbol{e}}_{r}, \widehat{\boldsymbol{e}}_{\theta}$, and $\widehat{\boldsymbol{e}}_{\varphi}$

$$
\begin{aligned}
& -\mathcal{A} \xi_{r}^{\prime}-\mathrm{i} \mathcal{B} \sin \theta \xi_{\varphi}^{\prime}=-\partial_{r} W^{\prime}-\frac{\rho^{\prime}}{\bar{\rho}} \bar{g}-\Pi^{\prime} \frac{\partial_{r} \bar{\rho}}{\bar{\rho}^{2}}, \\
& -\mathcal{A} \xi_{\theta}^{\prime}-\mathrm{i} \mathcal{B} \cos \theta \xi_{\varphi}^{\prime}=-\frac{1}{r} \partial_{\theta} W^{\prime}, \text { and } \\
& -\mathcal{A} \xi_{\varphi}^{\prime}+\mathrm{i} \mathcal{B} \cos \theta \xi_{\theta}^{\prime}+\mathrm{i} \mathcal{B} \sin \theta \xi_{r}^{\prime}=-\frac{\mathrm{i} m W^{\prime}}{r \sin \theta} .
\end{aligned}
$$

Here $\sigma_{\mathrm{M}}=\sqrt{\mathcal{A}}$ can be seen as a modified local wave angular velocity that corresponds to the modification of $\sigma_{\mathrm{s}}$ by the presence of the magnetic field. Thus, waves are only propagating if $\mathcal{A}>0$; in the other case, they are trapped in the vertical direction (cf. Schatzman 1993a; and Barnes et al. 1998) because the azimuthal magnetic field acts as a filter in this direction (see in Figs. 6 and 7). Moreover, we define

$W(\boldsymbol{r}, t)=\frac{\Pi}{\bar{\rho}}+\widetilde{\Phi}$,

which is the sum of the total wave dynamical pressure fluctuation $^{2}$ and of the gravific potential fluctuation.

Next, the continuity equation is given by

$\rho^{\prime}+\frac{1}{r^{2}} \partial_{r}\left(r^{2} \bar{\rho} \xi_{r}^{\prime}\right)+\frac{\bar{\rho}}{r}\left[\frac{1}{\sin \theta} \partial_{\theta}\left(\sin \theta \xi_{\theta}^{\prime}\right)+\frac{\mathrm{i} m \xi_{\varphi}^{\prime}}{\sin \theta}\right]=0$.

Afterwards, the adiabatic energy transport equation becomes

$\left(\frac{P^{\prime}}{\Gamma_{1} \bar{P}}-\frac{\rho^{\prime}}{\bar{\rho}}\right)+\frac{N^{2}}{\bar{g}} \xi_{r}^{\prime}=0$,

where the gravity and the Brunt-Väisälä frequency are given by $\bar{g}=|\mathrm{d} \bar{\Phi} / \mathrm{d} r|$ and $N^{2}=-\bar{g}\left(\mathrm{~d} \ln \bar{\rho} / \mathrm{d} r-1 / \Gamma_{1} \cdot \mathrm{d} \ln \bar{P} / \mathrm{d} r\right)$, respectively.

Finally, the Poisson's equation is written as

$\frac{1}{r^{2}} \partial_{r}\left(r^{2} \partial_{r} \Phi^{\prime}\right)+\frac{1}{r^{2}}\left[\frac{1}{\sin \theta} \partial_{\theta}\left(\sin \theta \partial_{\theta} \Phi^{\prime}\right)-\frac{m^{2}}{\sin ^{2} \theta} \Phi^{\prime}\right]=4 \pi G \rho^{\prime}$

From now on, we adopt the Cowling's approximation (Cowling 1941 ) where the wave's gravific potential fluctuation $(\widetilde{\Phi})$ is neglected. Therefore, Eq. (28) reduces to $W=\Pi / \bar{\rho}$.

\subsection{Local analysis}

Following Kumar et al. (1999) who studied the dynamics of lowfrequency magneto-gravito-inertial waves (cf. Appendix A), we now consider a more simplified set-up than ours to obtain their dispersion relation. Indeed, we consider a stellar radiation zone where both the angular velocity and the background magnetic field are uniform (i.e. $\Omega=\Omega_{\mathrm{s}}$ and $\boldsymbol{B}_{0}^{\mathrm{T}}=\overline{\boldsymbol{B}}$ ). Then, assuming

2 The dynamical total pressure $W$ is defined as $W=\Pi / \rho$. 
that all the perturbed quantities have a dependence proportional to $\exp [\mathrm{i}(\boldsymbol{k} \cdot \boldsymbol{r}+\sigma t)]$, the momentum equation (Eq. (2)) becomes

$$
-\bar{\rho} \sigma^{2} \boldsymbol{\xi}+\bar{\rho} 2 \mathrm{i} \sigma \boldsymbol{\Omega} \times \boldsymbol{\xi}+\bar{\rho} N^{2} \xi_{r} \widehat{\boldsymbol{e}}_{r}+\mathrm{i} \boldsymbol{k} \widetilde{P}=\frac{\mathrm{i}(\boldsymbol{k} \times \boldsymbol{b})}{\mu} \times \overline{\boldsymbol{B}} .
$$

After vectorial manipulations given in Appendix A, this yields to the dispersion relation for waves in this set-up (Eq. (A.6)):

$$
\begin{aligned}
& {\left[\sigma^{2}-(\widehat{\boldsymbol{B}} \cdot \boldsymbol{k})^{2} V_{\mathrm{A}}^{2}\right]^{2}-(\boldsymbol{N} \times \widehat{\boldsymbol{k}})^{2}\left[\sigma^{2}-(\widehat{\boldsymbol{B}} \cdot \boldsymbol{k})^{2} V_{\mathrm{A}}^{2}\right]} \\
& -4 \sigma^{2}(\boldsymbol{\Omega} \cdot \widehat{\boldsymbol{k}})^{2}=0,
\end{aligned}
$$

where $\widehat{\boldsymbol{B}}=\boldsymbol{B} / B, \boldsymbol{N}=N \widehat{\boldsymbol{e}}_{r}, \boldsymbol{\Omega}=\Omega_{\mathrm{s}} \widehat{\boldsymbol{e}}_{z}$, and $\widehat{\boldsymbol{k}}=\boldsymbol{k} / k$. This can be solved, leading to

$$
\begin{aligned}
\sigma^{2} & =(\widehat{\boldsymbol{B}} \cdot \boldsymbol{k})^{2} V_{\mathrm{A}}^{2}+\frac{1}{2}\left\{(\boldsymbol{N} \times \widehat{\boldsymbol{k}})^{2}+4(\boldsymbol{\Omega} \cdot \widehat{\boldsymbol{k}})^{2}\right. \\
& \left. \pm \sqrt{\left[(\boldsymbol{N} \times \widehat{\boldsymbol{k}})^{2}+4(\boldsymbol{\Omega} \cdot \widehat{\boldsymbol{k}})^{2}\right]^{2}+16(\widehat{\boldsymbol{B}} \cdot \boldsymbol{k})^{2} V_{\mathrm{A}}^{2}(\boldsymbol{\Omega} \cdot \widehat{\boldsymbol{k}})^{2}}\right\}
\end{aligned}
$$

Let us now discuss this dispersion relation as done by Heng \& Spitkovsky (2009). First, if we examine the non-rotating limit $\left(\Omega_{\mathrm{s}}=0\right)$, we get

$\sigma^{2}=\left\{\begin{array}{l}(\widehat{\boldsymbol{B}} \cdot \boldsymbol{k})^{2} V_{\mathrm{A}}^{2} \\ (\boldsymbol{N} \times \widehat{\boldsymbol{k}})^{2}+(\widehat{\boldsymbol{B}} \cdot \boldsymbol{k})^{2} V_{\mathrm{A}}^{2}\end{array}\right.$.

Two branches are then isolated: the low-frequency one corresponds to Alfvén waves while magneto-gravity waves, for which restoring forces are both buoyancy and Lorentz forces, are linked to the higher-frequency branch (see Schecter et al. 2001).

Then, if we examine the rotating system, both wave families couple through the Coriolis acceleration. On one hand, for lowfrequency Alfvén waves, Coriolis acceleration tries to balance the Lorentz force and for this reason, those waves are then called "magnetostrophic waves". Their approximate dispersion relation is given by

$$
\sigma^{2} \approx(\widehat{\boldsymbol{B}} \cdot \boldsymbol{k})^{2} V_{\mathrm{A}}^{2}\left(1-\frac{4(\boldsymbol{\Omega} \cdot \widehat{\boldsymbol{k}})^{2}}{(\boldsymbol{N} \times \widehat{\boldsymbol{k}})^{2}+4(\boldsymbol{\Omega} \cdot \widehat{\boldsymbol{k}})^{2}}\right) .
$$

On the other hand, magneto-gravity waves become magnetogravito-inertial waves, which are also called magneto-Poincaré waves and magneto-Rossby waves for those waves that are due to the star curvature and the associated variation of the local rotation on a tangent sphere. In this case, we obtain the following approximative dispersion relation:

$$
\begin{aligned}
\sigma^{2} \approx & (\boldsymbol{N} \times \widehat{\boldsymbol{k}})^{2}+4(\boldsymbol{\Omega} \cdot \widehat{\boldsymbol{k}})^{2} \\
& +(\widehat{\boldsymbol{B}} \cdot \boldsymbol{k})^{2} V_{\mathrm{A}}^{2}\left(1+\frac{4(\boldsymbol{\Omega} \cdot \widehat{\boldsymbol{k}})^{2}}{(\boldsymbol{N} \times \widehat{\boldsymbol{k}})^{2}+4(\boldsymbol{\Omega} \cdot \widehat{\boldsymbol{k}})^{2}}\right)
\end{aligned}
$$

Let us introduce the expansion of $\boldsymbol{k}$ both in the spherical and cylindrical coordinates:

$\boldsymbol{k}=k_{V} \widehat{\boldsymbol{e}}_{r}+\boldsymbol{k}_{H} \quad$ and $\quad \boldsymbol{k}=\boldsymbol{k}_{P}+k_{z} \widehat{\boldsymbol{e}}_{z}$,

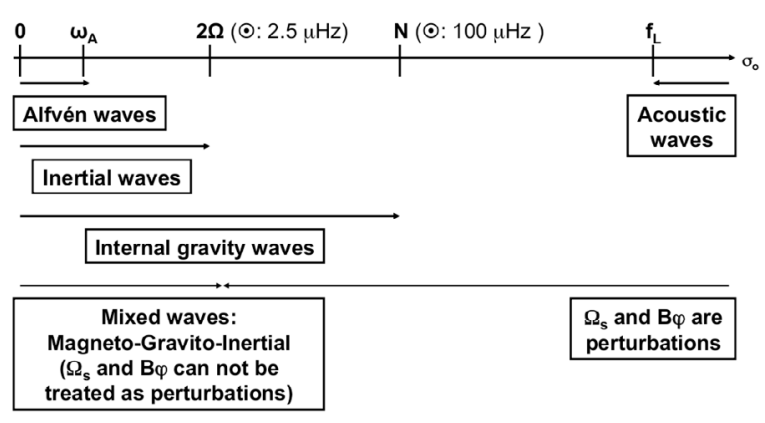

Fig. 2. Wave types in a rotating and magnetic stellar radiation zone and associated frequencies (where $f_{\mathrm{L}}$ is the Lamb's frequency). Values for the Sun $(\odot)$ at the bottom of the Tachocline are given in brackets.

where $\boldsymbol{k}_{H}=k_{\theta} \widehat{\boldsymbol{e}}_{\theta}+k_{\varphi} \widehat{\boldsymbol{e}}_{\varphi}$ and $\boldsymbol{k}_{P}=k_{\mathrm{S}} \widehat{\boldsymbol{e}}_{\mathrm{s}}+k_{\varphi} \widehat{\boldsymbol{e}}_{\varphi}$, with $(s, \varphi, z)$ the cylindrical coordinates and $\left\{\widehat{\boldsymbol{e}}_{j}\right\}_{j=s, \varphi, z}$ the associated unit-vector basis. Then, the dispersion relation is written as

$$
\begin{aligned}
\sigma^{2} \approx & N^{2} \frac{k_{H}^{2}}{k^{2}}+4 \Omega_{\mathrm{s}}^{2} \frac{k_{z}^{2}}{k^{2}} \\
& +(\widehat{\boldsymbol{B}} \cdot \boldsymbol{k})^{2} V_{\mathrm{A}}^{2}\left(1+\frac{4(\boldsymbol{\Omega} \cdot \widehat{\boldsymbol{k}})^{2}}{(\boldsymbol{N} \times \widehat{\boldsymbol{k}})^{2}+4(\boldsymbol{\Omega} \cdot \widehat{\boldsymbol{k}})^{2}}\right) .
\end{aligned}
$$

In the case of highly stratified stellar radiation zones where $\omega_{\mathrm{A}} \ll N$ and $2 \Omega_{\mathrm{s}} \ll N$, this reduces for low-frequency waves $(\sigma \ll N)$ to

$\frac{k_{H}^{2}}{k^{2}} \approx 0$,

which leads to the following hierarchy

$\left|k_{H}\right| \ll\left|k_{V}\right|$ and $\left|\xi_{V}\right| \ll\left|\xi_{H}\right|$

because of the anelastic approximation $\boldsymbol{\nabla} \cdot(\bar{\rho} \boldsymbol{\xi}) \approx 0$ where acoustic waves are filtered, which becomes in the local analysis case $\boldsymbol{k} \cdot \boldsymbol{\xi} \approx 0$.

From now on, we focus on this type of wave (in the case where $\omega_{\mathrm{A}} \ll N$ and $2 \Omega_{\mathrm{s}} \ll N$ ) for which $\sigma$ can be of the same order of magnitude as $\omega_{\mathrm{A}}$ and $2 \Omega_{\mathrm{s}}$, and the ratio $\omega_{\mathrm{A}} / 2 \Omega_{\mathrm{s}}$ is taken as general as possible (see Fig. 2 below).

\section{Low-frequency waves in rotating magnetic stellar radiation zones}

\subsection{The traditional approximation}

We first discuss the non-magnetic case, namely the gravitoinertial waves. In the general case, the Poincaré operator, which governs the spatial structure of waves, is of mixed type (elliptic and hyperbolic) and not separable (see the detailed discussions in Friedlander \& Siegman 1982; Dintrans et al. 1999; Dintrans \& Rieutord 2000). This leads to the appearance of detached shear layers associated with the underlying singularities of the adiabatic problem that could be crucial for transport and mixing processes in stellar radiation zones, since they are the seat of 
strong dissipation (cf. Stewartson \& Richard 1969; Stewartson \& Walton 1976; Dintrans et al. 1999; and Dintrans \& Rieutord 2000).

Let us now focus on the case of a uniform rotation $(\Omega=$ $\left.\Omega_{\mathrm{s}}\right)$. In the largest part of stellar radiation zones, we are in a regime where $2 \Omega_{\mathrm{s}} \ll N$. Since we are interested here in lowfrequency waves $(\sigma \ll N)$, the traditional approximation (hereafter TA), which consists in neglecting the latitudinal component of the rotation vector $\left(\Omega_{\mathrm{s}} \widehat{\boldsymbol{e}}_{z}\right),-\Omega_{\mathrm{s}} \sin \theta \widehat{\boldsymbol{e}}_{\theta}$, in the Coriolis acceleration, can be adopted in the super-inertial regime where $2 \Omega_{\mathrm{s}}<\sigma \ll N$ (see e.g. Eckart 1960; for a modern description in a stellar context see Bildsten et al. 1996; Lee \& Saio 1997; and Mathis et al. 2008). Then, variable separation in radial and horizontal eigenfunctions remains possible (cf. Friedlander 1987a), which corresponds to the ergodic (regular) elliptic gravito-inertial mode family (the $\mathrm{E}_{1}$ modes in Dintrans et al. 1999; and Dintrans \& Rieutord 2000). This approximation has to be carefully used however, because it changes the nature of the Poincare operator, and removes the singularities and associated shear layers that appear. Then, in the subinertial regime, where $\sigma \leq 2 \Omega_{\mathrm{s}}$, which corresponds to the equatorially trapped hyperbolic modes (the $\mathrm{H}_{2}$ modes in Dintrans et al. 1999; and Dintrans \& Rieutord 2000), the TA fails to reproduce the waves behaviour and the complete momentum equation has to be solved (see detailed examples in Gerkema \& Shrira 2005; and Gerkema et al. 2008). Note also the work by Fruman (2009), who examines the validity of the TA on the equatorial $\beta$-plane in function of the $2 \Omega_{\mathrm{s}} / N$ value.

As a first step, we thus studied the regular elliptic gravitoinertial waves for which the TA applies (Mathis et al. 2008; and Mathis 2009). In the MHD case, our purpose is to generalize the TA to i $\mathcal{B} \widehat{\boldsymbol{e}}_{z} \times \boldsymbol{\xi}$, thus neglecting the latitudinal component of $\widehat{\boldsymbol{e}}_{z}$ since $2 \Omega_{\mathrm{s}} \ll N, \omega_{\mathrm{A}} \ll N$, and $\sigma \ll N$. Therefore, we restrict ourselves here to the regular low-frequency waves for which the TA can be used as we show it in the next section. Its application domain is discussed in Sect. 4.

\subsection{Dynamical equations}

Assuming the TA for low-frequency magneto-gravito-inertial waves, we respectively get for the linearized momentum equation components:

$$
\begin{aligned}
& -\mathcal{A} \xi_{r}^{\prime}=-\partial_{r} W^{\prime}-N^{2} \xi_{r}^{\prime}-\frac{P^{\prime}}{\Gamma_{1} \bar{P}} \bar{g}-\Pi^{\prime} \frac{\partial_{r} \bar{\rho}}{\bar{\rho}^{2}}, \\
& -\mathcal{A} \xi_{\theta}^{\prime}-\mathrm{i} \mathcal{B} \cos \theta \xi_{\varphi}^{\prime}=-\frac{1}{r} \partial_{\theta} W^{\prime}, \\
& -\mathcal{A} \xi_{\varphi}^{\prime}+\mathrm{i} \mathcal{B} \cos \theta \xi_{\theta}^{\prime}=-\frac{i m W^{\prime}}{r \sin \theta},
\end{aligned}
$$

where the linearized energy equation (Eq. (30)) has been used to express the buoyancy force $-\frac{\rho^{\prime}}{\bar{\rho}} \bar{g}$.

We need to describe the different assumptions for using the TA. On one hand, since $\omega_{\mathrm{A}} \ll N$ and $2 \Omega_{\mathrm{s}} \ll N$, we neglect the $-\mathrm{i} \mathcal{B} \sin \theta \xi_{\varphi}^{\prime}$ term in the radial component of the momentum equation (Eq. (25)). On the other hand, the first term $-\mathcal{A} \xi_{r}^{\prime}$ also has to be neglected, but we first conserve it to make the historical link with works in the non-rotating and non-magnetic cases (Press 1981; Schatzman 1993b; Zahn et al. 1997) and those about gravito-inertial waves (Mathis et al. 2008; Mathis 2009).



Fig. 3. Inverse of the Rossby number $\left(v_{\mathrm{s}}\right)$ as a function of the frequency $(\sigma / 2 \pi)$ for axisymmetric waves $(m=0)$. We assume that $\Omega_{\mathrm{s}} / 2 \pi=$ $430 \mathrm{nHz}$. In the purely hydrodynamical case (namely those of gravitoinertial waves), the TA is allowed in the super-inertial regime where $2 \Omega_{\mathrm{s}}<\sigma$ (i.e. $\left.v_{\mathrm{s}}<1\right)$.

Finally, since $\xi_{r}^{\prime} \ll\left\{\xi_{\theta}^{\prime}, \xi_{\varphi}^{\prime}\right\}$ (cf. Eq. (41)) for low-frequency internal waves, the term $+\mathrm{i} \mathcal{B} \sin \theta \xi_{r}^{\prime}$ is neglected in the azimuthal component (Eq. (27)).

\subsection{Spatial structure of the wave-displacement, velocity, and magnetic field}

\subsubsection{Spatial structure of the horizontal components of the displacement}

After successively eliminating $\xi_{\theta}^{\prime}$ and $\xi_{\varphi}^{\prime}$ between Eqs. (43) and (44), each of them is expressed in function of $W^{\prime}=$ $1 / \bar{\rho}\left[P^{\prime}+\left(\boldsymbol{B}_{0} \cdot \boldsymbol{b}^{\prime}\right) / \mu\right]$ as

$\xi_{\theta}^{\prime}=\frac{1}{r \sigma_{\mathrm{M}}^{2}} \frac{1}{\left(1-v_{\mathrm{M}}^{2} x^{2}\right) \sqrt{1-x^{2}}}\left[-\left(1-x^{2}\right) \partial_{x}+m v_{\mathrm{M}} x\right] W^{\prime}$,

$\xi_{\varphi}^{\prime}=\mathrm{i} \frac{1}{r \sigma_{\mathrm{M}}^{2}} \frac{1}{\left(1-v_{\mathrm{M}}^{2} x^{2}\right) \sqrt{1-x^{2}}}\left[-v_{\mathrm{M}} x\left(1-x^{2}\right) \partial_{x}+m\right] W^{\prime}$,

where $x=\cos \theta$ has been introduced. Then $v_{M}$ is defined as

$v_{\mathrm{M}}=\mathcal{B} \mathcal{A}^{-1}=v_{\mathrm{s}}\left(\sigma_{\mathrm{s}}, \Omega_{\mathrm{s}}\right) F_{\mathrm{M}}\left(\sigma_{\mathrm{s}}, \Omega_{\mathrm{s}}, \omega_{\mathrm{A}}\right)$,

where

$v_{\mathrm{s}}=\frac{2 \Omega_{\mathrm{s}}}{\sigma_{\mathrm{s}}}=R_{\mathrm{o}}^{-1} \quad$ and $\quad F_{\mathrm{M}}=\frac{1-m \Lambda_{\mathrm{E}}}{1-\frac{m^{2}}{2} v_{\mathrm{s}} \Lambda_{\mathrm{E}}}$.

Here, $v_{\mathrm{S}}$ is the spin parameter (Fig. 3), which equals the inverse of the Rossby number $R_{\mathrm{o}}=\sigma_{\mathrm{s}} / 2 \Omega_{\mathrm{s}}$ (cf. Lee \& Saio 1997), and $F_{\mathrm{M}}=v_{\mathrm{M}} / v_{\mathrm{s}}$ gives its modification by the magnetic field.

We introduce the wave's Elsasser number

$\Lambda_{\mathrm{E}}=\frac{\omega_{\mathrm{A}}^{2}}{\left(\Omega_{\mathrm{s}} \sigma_{\mathrm{s}}\right)}$,

which gives the ratio of the Lorentz force with the Coriolis acceleration (cf. Eqs. (13) and (15) and Fig. 4) ${ }^{3}$.

${ }^{3}$ Since $\mathcal{A}>0$, then $1-\frac{m^{2}}{2} v_{\mathrm{s}} \Lambda_{E}>0$. 


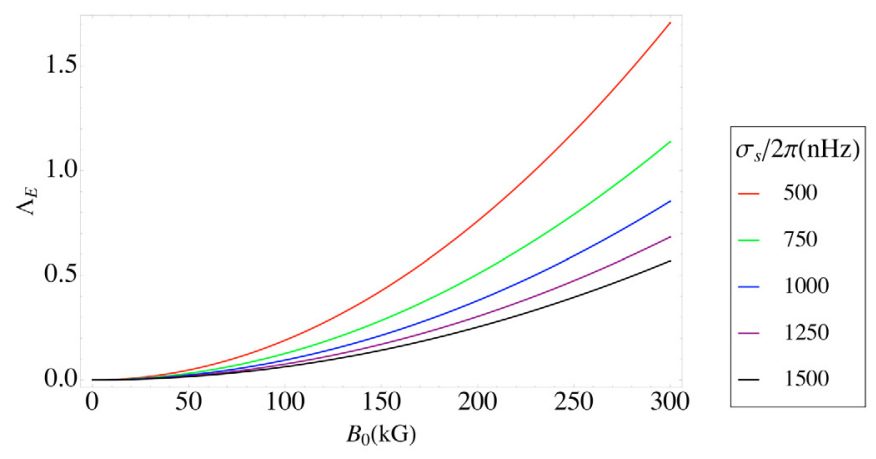

Fig. 4. Elsasser number $\left(\Lambda_{\mathrm{E}}\right)$ as a function of the wave frequency in the corotating frame $\left(\sigma_{\mathrm{s}}\right)$ and of the azimuthal magnetic field amplitude at the equator $(\theta=\pi / 2)$ just below the solar tachocline ( $B_{0}=\sqrt{\mu \bar{\rho}} R_{T} \omega_{\mathrm{A}}$, where $R_{T} \approx 0.71 R_{\odot}, R_{\odot}$ being the solar radius).

There $\bar{\rho}=2 \mathrm{~g} \mathrm{~cm}^{-3}$ and we assume that $\Omega_{\mathrm{s}} / 2 \pi=430 \mathrm{nHz}$. The upper limit for the amplitude of the magnetic field has been chosen following constraints obtained by Antia et al. (2003).

\subsubsection{Spatial structure of the radial component of the displacement and of the pressure}

As in the non-magnetic case and because of the equation structure (cf. Lee \& Saio 1997; Mathis et al. 2008), we choose to expand scalar quantities and the displacement's vertical component as

$X^{\prime}=\sum_{k} X_{k, m}^{\prime}(r) \Theta_{k, m}\left(\cos \theta ; v_{\mathrm{M}}\right)$

$\xi_{r}^{\prime}=\sum_{k} \xi_{r ; k, m}^{\prime}(r) \Theta_{k, m}\left(\cos \theta ; v_{\mathrm{M}}\right)$

The $\Theta_{k, m}$ functions are the orthogonal Hough functions for which $\int_{-1}^{1} \Theta_{k_{1}, m}\left(x ; v_{\mathrm{M}}\right) \Theta_{k_{2}, m}\left(x ; v_{\mathrm{M}}\right) \mathrm{d} x=C_{k_{1}, m}\left(v_{\mathrm{M}}\right) \delta_{k_{1}, k_{2}}$ where $\delta$ is the Kronecker symbol (Laplace 1799; Hough 1898; Longuet-Higgins 1968; Lindzen \& Chapman 1969). They are the eigenfunctions (with the associated eigenvalues $\Lambda_{k, m}$ ) of the so-called "Laplace tidal equation" (hereafter LTE):

$\mathcal{L}_{v_{\mathrm{M}}}\left[\Theta_{k, m}\left(x ; v_{\mathrm{M}}\right)\right]=-\Lambda_{k, m}\left(v_{\mathrm{M}}\right) \Theta_{k, m}\left(x ; v_{\mathrm{M}}\right)$,

where the Laplace tidal operator is given by

$$
\begin{aligned}
\mathcal{L}_{v_{\mathrm{M}}} \equiv & \frac{\mathrm{d}}{\mathrm{d} x}\left(\frac{1-x^{2}}{1-v_{\mathrm{M}}^{2} x^{2}} \frac{\mathrm{d}}{\mathrm{d} x}\right) \\
& -\frac{1}{1-v_{\mathrm{M}}^{2} x^{2}}\left(\frac{m^{2}}{1-x^{2}}+m v_{\mathrm{M}} \frac{1+v_{\mathrm{M}}^{2} x^{2}}{1-v_{\mathrm{M}}^{2} x^{2}}\right)
\end{aligned}
$$

For a detailed discussion of the boundary conditions of the LTE, we refer the reader to the Lee \& Saio work (1997). Examples of Hough functions are given in Fig. 5.

As emphasized in Mathis et al. (2008), pro and retrograde eigenfunctions (and eigenvalues) are different. This crucial point for induced angular momentum transport will be discussed in detail in Paper II.
This allows separating the radial and latitudinal variables for $\xi_{\theta}^{\prime}$ and $\xi_{\varphi}^{\prime}$. Introducing the amplitude of the horizontal displacement $\boldsymbol{\xi}_{H}=\xi_{\theta} \widehat{e}_{\theta}+\xi_{\varphi} \widehat{e}_{\varphi}$ leads to

$\xi_{H ; k, m}^{\prime}(r)=\frac{1}{r \sigma_{\mathrm{M}}^{2}} W_{k, m}^{\prime}$,

and the latitudinal component of $\xi^{\prime}$ is written

$\xi_{\theta ; k, m}^{\prime}=\xi_{H ; k, m}^{\prime} \mathcal{H}_{k, m}^{\theta}\left(x ; v_{\mathrm{M}}\right)$,

where

$\mathcal{H}_{k, m}^{\theta}\left(x ; v_{\mathrm{M}}\right)=\mathcal{L}_{v_{\mathrm{M}} ; m}^{\theta}\left[\Theta_{k, m}\left(x ; v_{\mathrm{M}}\right)\right]$

with

$\mathcal{L}_{v_{\mathrm{M}} ; m}^{\theta} \equiv \frac{1}{\left(1-v_{\mathrm{M}}^{2} x^{2}\right) \sqrt{1-x^{2}}}\left[-\left(1-x^{2}\right) \frac{\mathrm{d}}{\mathrm{d} x}+m v_{\mathrm{M}} x\right]$.

In the same way, its azimuthal component is given by

$\xi_{\varphi ; k, m}^{\prime}=\mathrm{i} \xi_{H ; k, m}^{\prime} \mathcal{H}_{k, m}^{\varphi}\left(x ; v_{\mathrm{M}}\right)$

where

$\mathcal{H}_{k, m}^{\varphi}\left(x ; v_{\mathrm{M}}\right)=\mathcal{L}_{v_{\mathrm{M}} ; m}^{\varphi}\left[\Theta_{k, m}\left(x ; v_{\mathrm{M}}\right)\right]$

with

$\mathcal{L}_{v_{\mathrm{M}} ; m}^{\varphi} \equiv \frac{1}{\left(1-v_{\mathrm{M}}^{2} x^{2}\right) \sqrt{1-x^{2}}}\left[-v_{\mathrm{M}} x\left(1-x^{2}\right) \frac{\mathrm{d}}{\mathrm{d} x}+m\right]$

Then, the radial component of the momentum equation assuming the TA (Eq. (42)), the continuity equation (Eq. (29)), and the energy transport equation (Eq. (30)) become

$-\sigma_{\mathrm{M}}^{2} \xi_{r ; k, m}^{\prime}=-\partial_{r} W_{k, m}^{\prime}-\frac{\rho_{k, m}^{\prime}}{\bar{\rho}} \bar{g}-\Pi_{k, m}^{\prime} \frac{\partial_{r} \bar{\rho}}{\bar{\rho}^{2}}$

$\rho_{k, m}^{\prime}+\frac{1}{r^{2}} \frac{\mathrm{d}}{\mathrm{d} r}\left(r^{2} \bar{\rho} \xi_{r ; k, m}^{\prime}\right)-\frac{\bar{\rho}}{r} \Lambda_{k, m}\left(v_{\mathrm{M}}\right) \xi_{H ; k, m}^{\prime}=0$,

$\frac{\rho_{k, m}^{\prime}}{\bar{\rho}}=\frac{1}{\Gamma_{1}} \frac{P_{k, m}^{\prime}}{\bar{P}}+\frac{N^{2}}{\bar{g}} \xi_{r ; k, m}^{\prime}$

Eliminating $\rho_{k, m}^{\prime}$ between the previous equations and assuming the Cowling's approximation, we get the following system for $r^{2} \xi_{r ; k, m}$ and $W_{k, m}^{\prime}$ :

$$
\begin{aligned}
\frac{\mathrm{d} W_{k, m}^{\prime}}{\mathrm{d} r}= & \frac{N^{2}}{\bar{g}} W_{k, m}^{\prime}+\frac{1}{r^{2}}\left(\sigma_{\mathrm{M}}^{2}-N^{2}\right)\left(r^{2} \xi_{r ; k, m}^{\prime}\right) \\
& -\frac{1}{\Gamma_{1}} \frac{\mathrm{d} \ln \bar{P}}{\mathrm{~d} r} \frac{P_{\mathrm{M} ; k, m}^{\prime}}{\bar{\rho}},
\end{aligned}
$$

$$
\begin{aligned}
\frac{\mathrm{d}}{\mathrm{d} r}\left(r^{2} \xi_{r ; k, m}^{\prime}\right)= & {\left[\frac{\Lambda_{k, m}\left(\nu_{\mathrm{M}}\right)}{\sigma_{\mathrm{M}}^{2}}-\frac{\bar{\rho} r^{2}}{\Gamma_{1} \bar{P}}\right] W_{k, m}^{\prime}-\frac{1}{\Gamma_{1} \bar{P}} \frac{\mathrm{d} \bar{P}}{\mathrm{~d} r}\left(r^{2} \xi_{r ; k, m}^{\prime}\right) } \\
& +\frac{r^{2}}{\Gamma_{1} \bar{P}} P_{\mathrm{M} ; k, m}^{\prime}
\end{aligned}
$$



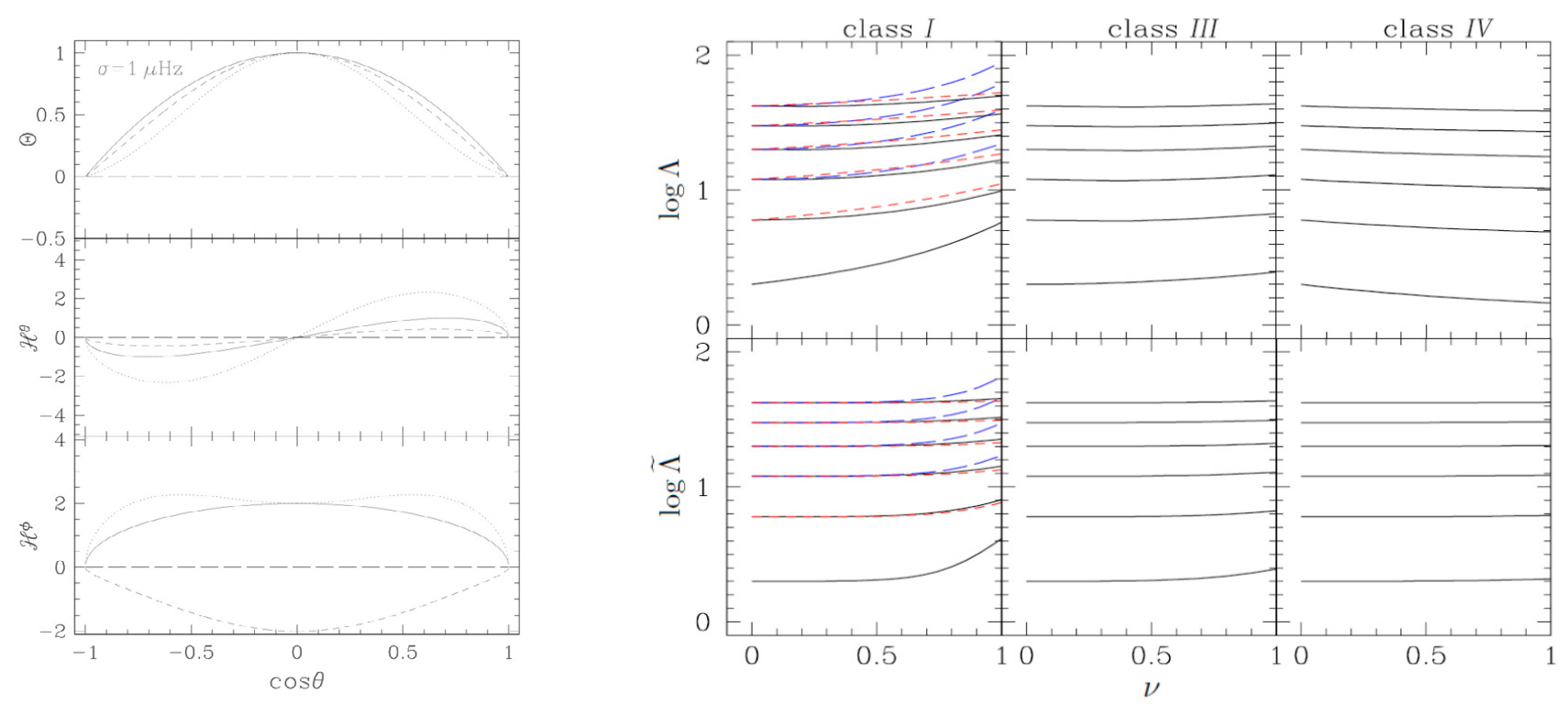

Fig. 5. Left: hough functions $\Theta_{k, m}(\cos \theta ; v)($ Top $)$ and associated latitudinal $\mathcal{H}_{k, m}^{\theta}(\cos \theta ; v)$ and azimuthal $\mathcal{H}_{k, m}^{\varphi}(\cos \theta ; v)$ functions $($ Middle and Bottom) $m=2, v=0$ (continuous lines); $m=2, v=0.86$ (dotted lines); $m=-2, v=0.86$ (dashed lines). Right: (Top) Eigenvalue ( $\Lambda$ ) of Laplace's tidal equation (in the presence of rotation and of magnetic field) in the range relevant for a solar model and the MHD TA $(v<1)$. (Bottom) Equivalent horizontal eigenvalue $(\widetilde{\Lambda})$ (see Eq. (85)). (Left) Class I waves with $1 \leq l \leq 6$, and $m=-l+2$ (black continuous line), $m=0$ (long blue dashed line), and $m=l$ (dashed red line). (Middle) Class III waves ( $s=0, s$ being defined in Eq. (89)) have negative values of $m(m=0, \cdots,-5)$. (Right) Class IV waves $(s=-1)$ have indices $m=-1, \cdots,-6$. Waves classes are discussed in Sect. 4.2. (see also Mathis et al. 2008).

where we explicitly introduce the wave magnetic pressure expansion

$$
\begin{aligned}
\widetilde{P}_{\mathrm{M}} & =\frac{\left(\boldsymbol{B}_{0}^{\mathrm{T}} \cdot \boldsymbol{b}\right)}{\mu} \\
& =\sum_{\sigma, m, k}\left\{P_{\mathrm{M} ; k, m}^{\prime}(r) \Theta_{k, m}\left(\cos \theta ; v_{\mathrm{M}}\right) \exp (\mathrm{i} m \varphi) \exp (\mathrm{i} \sigma t)\right\}
\end{aligned}
$$

Terms where it explicitly appears are introduced through $P_{k, m}^{\prime}$ in the energy equation (Eq. (63)) because of Eq. (14).

This is a generalization of the system obtained by Press (1981) in the non-rotating and non-magnetic case where $\sigma_{\mathrm{M}}$ and $\Lambda_{k, m}\left(v_{\mathrm{M}}\right)$ respectively replace $\sigma$ and $l(l+1)$, where $l$ is the orbital number of the classical spherical harmonics.

Adopting the anelastic approximation, where magnetoacoustic waves are filtered out (i.e. $\boldsymbol{\nabla} \cdot(\bar{\rho} \boldsymbol{\xi})=0$ and the terms proportional to $c_{\mathrm{s}}^{-2}$ are neglected, where $c_{\mathrm{s}}=\sqrt{\frac{\Gamma_{1} \bar{P}}{\bar{\rho}}}$ is the sound speed), and following the procedure given in Zahn (1970, 1975) and in Press (1981), we respectively get for the vertical displacement

$$
\frac{\mathrm{d}^{2} \Psi_{k, m}}{\mathrm{~d} r^{2}}+\left[\left(\frac{N^{2}}{\sigma_{\mathrm{M}}^{2}}-1\right) \frac{\Lambda_{k, m}\left(v_{\mathrm{M}}\right)}{r^{2}}\right] \Psi_{k, m}=0
$$

where $\Psi_{k, m}=\bar{\rho}^{1 / 2} r^{2} \xi_{r ; k, m}^{\prime}$, and for the pressure fluctuation

$\frac{\mathrm{d}^{2} \mathcal{W}_{k, m}}{\mathrm{~d} r^{2}}+\left[\left(\frac{N^{2}}{\sigma_{\mathrm{M}}^{2}}-1\right) \frac{\Lambda_{k, m}\left(v_{\mathrm{M}}\right)}{r^{2}}\right] \boldsymbol{W}_{k, m}=0$,

where $\mathcal{W}_{k, m}=\left[\left(\bar{\rho} r^{2}\right) / N^{2}\right]^{1 / 2} W_{k, m}^{\prime}$.

\subsubsection{The final wave-displacement, velocity, and magnetic field}

Once one solves Eq. (67) or Eq. (68), the associated final expression for $\boldsymbol{\xi}, \boldsymbol{u}$ and $\boldsymbol{b}$ are derived. First, using Eqs. $((51)-(45)-(46)-(54))$, the wave-displacement is given by

$\boldsymbol{\xi}=\sum_{j=\{r, \theta, \varphi\}}\left[\sum_{\sigma, m, k} \xi_{j ; k, m}\right] \widehat{\boldsymbol{e}}_{j}$

where

$\xi_{r ; k, m}=\xi_{r ; k, m}^{\prime} \Theta_{k, m}\left(\cos \theta ; \nu_{\mathrm{M}}\right) \exp (\mathrm{i} m \varphi) \exp (\mathrm{i} \sigma t)$,

$\xi_{\theta ; k, m}=\frac{1}{r \sigma_{\mathrm{M}}^{2}} W_{k, m}^{\prime} \mathcal{H}_{k, m}^{\theta}\left(\cos \theta ; v_{\mathrm{M}}\right) \exp (\mathrm{i} m \varphi) \exp (\mathrm{i} \sigma t)$,

$\xi_{\varphi ; k, m}=\mathrm{i} \frac{1}{r \sigma_{\mathrm{M}}^{2}} W_{k, m}^{\prime} \mathcal{H}_{k, m}^{\varphi}\left(\cos \theta ; v_{\mathrm{M}}\right) \exp (\mathrm{i} m \varphi) \exp (\mathrm{i} \sigma t)$.

Then, the wave velocity field and magnetic field are straightforwardly derived using $\boldsymbol{u}=\mathrm{i} \sigma_{\mathrm{s}} \boldsymbol{\xi}$ and $\boldsymbol{b}=\mathrm{i} m \sqrt{\mu \bar{\rho}} \omega_{\mathrm{A}} \boldsymbol{\xi}$ (cf. Eqs. (21) and (22)).

Finally, the dynamical pressure is given by

$\Pi=\sum_{\sigma, m, k} \Pi_{k, m}^{\prime} \Theta_{k, m}\left(\cos \theta ; v_{\mathrm{M}}\right) \exp (\mathrm{i} m \varphi) \exp (\mathrm{i} \sigma t)$,

where $\Pi_{k, m}^{\prime}=\bar{\rho} W_{k, m}^{\prime}$.

Therefore, one of the main important conclusions of the study of this model, which assumes uniform angular velocity $\left(\Omega_{\mathrm{s}}\right)$ and Alfvén frequency $\left(\omega_{\mathrm{A}}\right)$, is that the wave-induced perturbations in this case can be completely expressed as a function of the Hough functions used in the non-magnetic uniformly 
rotating case. In that framework, the spin parameter $\left(v_{\mathrm{s}}\right)$ is now replaced by $v_{\mathrm{M}}$, which accounts for magnetic field. The simplest case of magneto-gravity waves is treated in Appendix B.

However, we have to emphasize that such a neat variable separation is not possible in the case of a general differential rotation and azimuthal magnetic field $\left(\Omega(r, \theta), B_{\varphi}(r, \theta)=\right.$ $\left.\sqrt{\mu \rho} r \sin \theta \omega_{\mathrm{A}}(r, \theta)\right)$, even if the TA applies (cf. Mathis 2009). In that case, which is beyond the scope of the present paper, a more subtle treatment of the dynamical equations has to be adopted.

\subsubsection{Asymptotic analysis}

Let us now consider the low-frequency waves for which $\sigma \ll N$. We introduce the vertical wave number

$k_{V ; k, m}(r) \approx\left(\frac{N}{\sigma_{\mathrm{M}}}\right) \frac{\Lambda_{k, m}^{1 / 2}\left(v_{\mathrm{M}}\right)}{r} ;$

then, Eq. (67) is written

$\frac{\mathrm{d}^{2} \Psi_{k, m}}{\mathrm{~d} r^{2}}+k_{V ; k, m}^{2} \Psi_{k, m}=0$.

For these waves the J.W.K.B. approximation can be adopted (cf. Landau \& Lifshitz 1966; Press 1981; Schatzman 1993b; Zahn et al. 1997). Applying it to Eq. (75), we get ${ }^{4}$

$\xi_{r ; k, m}^{\prime}=E_{k, m}(r) \exp \left[\mathrm{i} \Phi_{k, m}(r)\right]$ with $\Phi_{k, m}(r)=\int_{r}^{r_{\mathrm{c}}} k_{V ; k, m} \mathrm{~d} r^{\prime}$,

where $r_{\mathrm{c}}$ is the radius of the convection-radiation border while the amplitude function is given by

$E_{k, m}=A_{k, m} r^{-3 / 2} \bar{\rho}^{-1 / 2}\left(\frac{N}{\sigma_{\mathrm{M}}}\right)^{-1 / 2}$.

Here, $A_{k, m}$ is the wave-displacement amplitude that has to be obtained using excitation models corresponding to the stochastic excitation by turbulent convective movements (Kiraga et al. 2003, 2005; Dintrans et al. 2005; Rogers \& Glatzmaier 2005, 2006; Belkacem et al. 2009) or to the $\kappa$-mechanism (Lee \& Saio 1987; Unno et al. 1989; Lee \& Baraffe 1995).

We then derive $\boldsymbol{\xi}, \boldsymbol{u}, \boldsymbol{b}$ and $\Pi$. First, we get

$\boldsymbol{\xi}=\sum_{j}\left[\sum_{\sigma, m, k} \xi_{j ; k, m}\right] \widehat{\boldsymbol{e}}_{j}$

where

$$
\begin{aligned}
\xi_{r ; k, m}= & E_{k, m}(r) \exp \left[\mathrm{i} \Phi_{k, m}(r)\right] \\
& \times \Theta_{k, m}\left(\cos \theta ; v_{\mathrm{M}}\right) \exp (\mathrm{i} m \varphi) \exp (\mathrm{i} \sigma t), \\
\xi_{\theta ; k, m}= & -\mathrm{i} \frac{r k_{V ; k, m}}{\Lambda_{k, m}\left(v_{\mathrm{M}}\right)} E_{k, m}(r) \exp \left[\mathrm{i} \Phi_{k, m}(r)\right] \\
& \times \mathcal{H}_{k, m}^{\theta}\left(\cos \theta ; v_{\mathrm{M}}\right) \exp (\mathrm{i} m \varphi) \exp (\mathrm{i} \sigma t), \\
\xi_{\varphi ; k, m}= & \frac{r k_{V ; k, m}}{\Lambda_{k, m}\left(v_{\mathrm{M}}\right)} E_{k, m}(r) \exp \left[\mathrm{i} \Phi_{k, m}(r)\right] \\
& \times \mathcal{H}_{k, m}^{\varphi}\left(\cos \theta ; v_{\mathrm{M}}\right) \exp (\mathrm{i} m \varphi) \exp (\mathrm{i} \sigma t) .
\end{aligned}
$$

\footnotetext{
${ }^{4}$ Here, we consider the case of solar-type stars for which the group velocity is negative (cf. Eq. (86)). In the case of massive stars, where it is positive, the phase function is $\exp \left[\mathrm{i} \int_{r_{\mathrm{c}}}^{r} k_{V ; k, m} \mathrm{~d} r^{\prime}\right]$.
}

Next, $\boldsymbol{u}$ and $\boldsymbol{b}$ are obtained once again using Eqs. (21) and (22). Finally, we get using Eqs. (61) and (63) in the anelastic approximation regime

$\Pi=\sum_{\sigma, m, k} \Pi_{k, m}^{\prime} \Theta_{k, m}\left(\cos \theta ; \nu_{\mathrm{M}}\right) \exp (\mathrm{i} m \varphi) \exp (\mathrm{i} \sigma t)$

with

$\Pi_{k, m}^{\prime}=-\mathrm{i} \bar{\rho} \frac{N^{2}}{k_{V ; k, m}} E_{k, m}(r) \exp \left[\mathrm{i} \Phi_{k, m}(r)\right]$.

On the other hand, following Pantillon et al. (2007), we can define a horizontal wave number given by

$k_{H ; k, m}(r)=\frac{\widetilde{\Lambda}_{k, m}^{1 / 2}\left(v_{\mathrm{M}}\right)}{r}$,

where (cf. Fig. 5)

$\widetilde{\Lambda}_{k, m}^{2}\left(v_{\mathrm{M}}\right)=\frac{\left\langle\left|r^{2} \nabla_{H}^{2} \Theta_{k, m}\left(\cos \theta ; v_{\mathrm{M}}\right)\right|^{2}\right\rangle_{\theta}}{\left\langle\left|\Theta_{k, m}\left(\cos \theta ; v_{\mathrm{M}}\right)\right|^{2}\right\rangle_{\theta}}$

$\langle\cdots\rangle_{\theta}=1 / 2 \int_{0}^{\pi} \cdots \sin \theta \mathrm{d} \theta$ and $\nabla_{H}^{2}=\frac{1}{\sin \theta} \partial_{\theta}\left(\sin \theta \partial_{\theta}\right)+\frac{1}{\sin ^{2} \theta} \partial \varphi^{2}$ is the horizontal spherical Laplacian.

In the absence of rotation and of magnetic field $\left(v_{M}=0\right)$, we recover $\widetilde{\Lambda}_{k, m}\left(v_{\mathrm{M}}\right)=\Lambda_{k, m}\left(v_{\mathrm{M}}\right)=l(l+1)$. The associated monochromatic vertical wave group velocity is given by

$V_{g ; k, m}=\frac{\mathrm{d} \sigma_{\mathrm{s}}}{\mathrm{d} k_{V ; k, m}}=-\frac{\sigma_{\mathrm{M}}}{\sigma_{\mathrm{s}}} \frac{\sigma_{\mathrm{M}}}{k_{V ; k, m}}=-\left(\frac{\sigma_{\mathrm{M}}}{\sigma_{\mathrm{s}}}\right)^{2} V_{p ; k, m}$,

where $V_{p ; k, m}=\frac{\sigma_{\mathrm{s}}}{k_{V ; k, m}}$ is the monochromatic wave phase velocity in solar-type stars. Signs are due to the direction of the excitation kinetic energy flux directed from the convective envelope to the star's center; in massive stars, signs are inverted. Finally, to determine eigenfrequencies one has to follow strictly the same methodology as described in Friedlander $(1987 a, b)$ and in Lee \& Saio (1989).

\section{The traditional approximation in the MHD case}

\subsection{Validity domain}

The MHD TA can be applied in spherical shells where

$\mathcal{D}\left(x ; v_{\mathrm{M}}\right)=1-v_{\mathrm{M}}^{2} x^{2}>0 \quad \forall x \in[-1,1]$,

thus as long $\left|v_{\mathrm{M}}\right|<1$ and $\mathcal{A}>0 \forall r$ and $\forall \theta \in[0, \pi]$ (cf. Fig. 6). In this regime, waves are elliptic and regular at all latitudes. In the other case, where both $\mathcal{D}>0$ and $\mathcal{D} \leq 0\left(\left|v_{\mathrm{M}}\right| \geq 1\right.$ and $\left.\mathcal{A}>0\right)$, waves become hyperbolic and trapped in an equatorial belt (see Dintrans \& Rieutord 2000, for example) where $\theta \in\left[\theta_{\mathrm{c}}, \pi-\theta_{\mathrm{c}}\right]$, with $\theta_{\mathrm{c}}$ the critical colatitude

$\theta_{\mathrm{c}}=\cos ^{-1}\left(\left|v_{\mathrm{M}}\right|^{-1}\right)$,

where $\mathcal{D}=0$ (cf. Fig. 7). Then, the adiabatic velocity field is singular and the MHD TA cannot be applied (see Sect. 3.1, and references therein), because the wave behaviour is possibly ruled by the dissipation in wave attractors (see for example in the hydrodynamical case Dintrans et al. 1999; Dintrans \& Rieutord 2000; and Maas \& Harlander 2007). This is a strict generalization of the criteria derived in the hydrodynamical case for gravito-inertial waves (Mathis et al. 2008; Mathis 2009). 

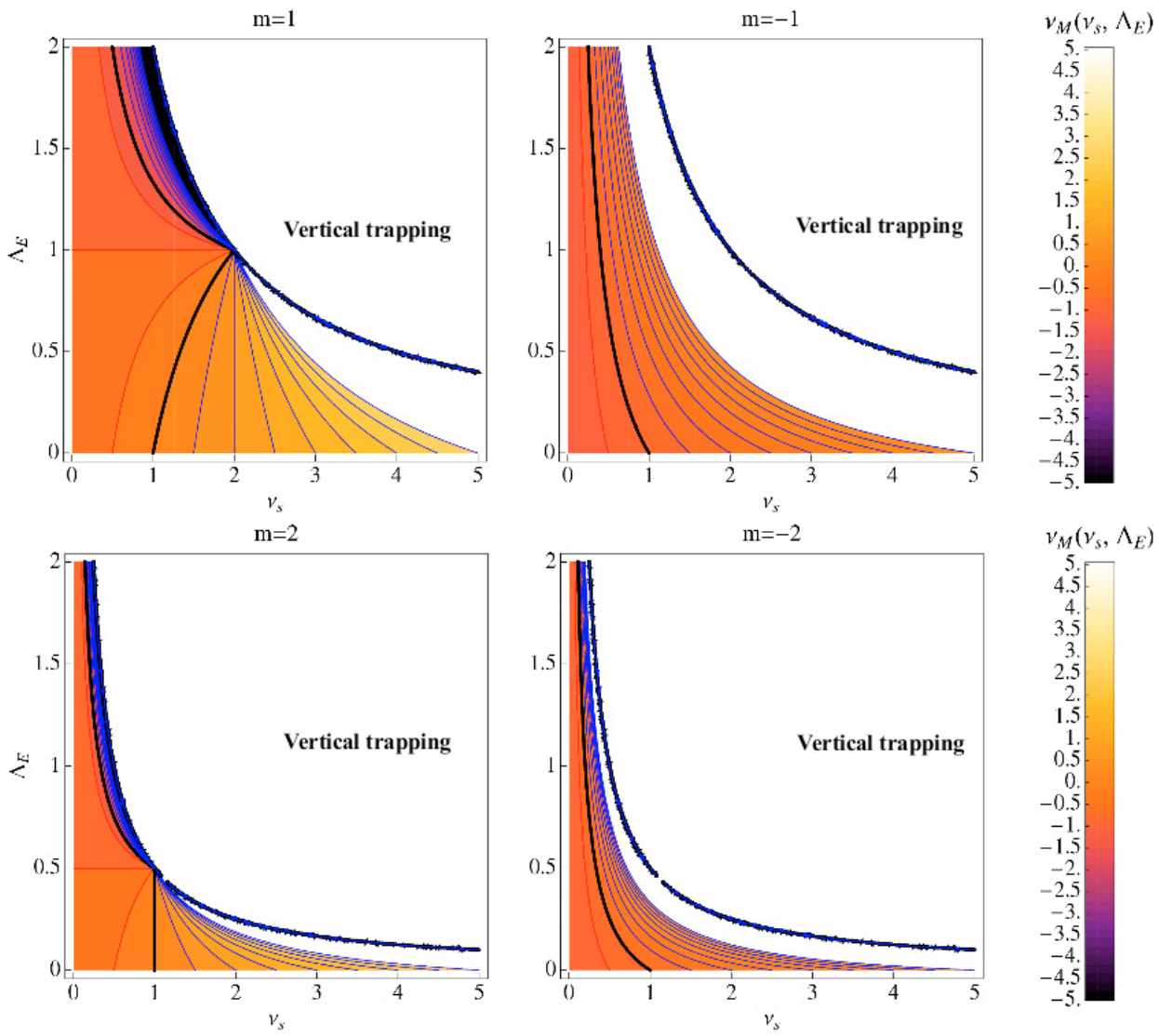

Fig. 6. Top: $v_{\mathrm{M}}$ as a function of $v_{\mathrm{s}}$ and $\Lambda_{\mathrm{E}}$ for non-axisymmetric retrograde $(m=1)$ and prograde $(m=-1)$ waves. The surfaces such that $\left|v_{\mathrm{M}}\right|=1$ are given by the thick black lines and the iso- $v_{M}$ lines for $\left|v_{M}\right|<1$ and $\left|v_{M}\right|>1$ are given by the red and the blue lines. The big external white region in the top-right corner in each case corresponds to the $\left(v_{\mathrm{s}}, \Lambda_{\mathrm{E}}\right)$ domain where $\mathcal{A} \leq 0$, the wave being thus vertically trapped there. The MHD TA applies when $\left|v_{\mathrm{M}}\right|<1$ and $\mathcal{A}>0$ (red lines). In the other case (blue and black lines, i.e. $\left|v_{\mathrm{M}}\right| \geq 1$ and $\mathcal{A}>0$ ), it does not apply due to the singularity at the critical latitude $\left(\theta_{\mathrm{c}}\right)$ given in Eq. (88) and plotted in Fig. (7). Bottom: same for $m=2$ and $m=-2$.

\subsection{Wave classification}

Under the MHD TA (as long as $\left|v_{M}\right|<1$ ), four types of magnetogravito-inertial waves can be identified (see Townsend 2003; and Mathis et al. 2008, and references therein in the hydrodynamical case).

- Class $I$ waves. They are internal gravity waves, which exist in the non-rotating and in the non-magnetic cases, which are modified both by Coriolis acceleration and the Lorentz force. Their eigenvalue $\left(\Lambda_{k, m}\right)$, hence their radial wave number, $k_{V ; k, m}(r) \equiv\left(N / \sigma_{\mathrm{M}}\right) \cdot \Lambda_{k, m}^{1 / 2}\left(v_{\mathrm{M}}\right) / r$, are increased. Such waves are also called magneto-Poincaré waves (see Zaqarashvili et al. 2009; Heng \& Spitovsky 2009 and Sect. 2).

- Class II waves. They are purely retrograde waves $(m>0)$, which only exist in the case of $v_{M}$ high values. Their dynamics are driven by the conservation of the specific vorticity combined with the effects of curvature and by the Lorentz force. However, since $\left|v_{\mathrm{M}}\right| \geq 1$ in this case, they cannot be treated using the MHD TA. In the hydrodynamical case, they are called "quasi-inertial" waves, which corresponds to the geophysical Rossby waves (see Provost et al. 1981). Such magneto-Rossby waves have recently been studied by Zaqarashvili et al. (2007), Zaqarashvili et al. (2009), and Heng \& Spitovsky (2009) in the limit of a thin stratified layer.
- Class III waves. These are mixed class I and class II waves, and $m \leq 0$ waves exist in the absence of rotation and of magnetic field. Waves with $m>0$ appear when $v_{M}=m+1$ with low eigenvalues, while their horizontal eigenfunctions are $\Theta_{k, m}\left(v_{\mathrm{M}}=m+1 ; x\right)=P_{m+1}^{m}(x)$. When they appear and have low eigenvalues, they behave mostly like class II waves; $m \leq 0$ and $m>0$ waves with high eigenvalues behave rather like class $I$ waves. Their eigenvalues are much lower than those of class $I$ waves. Thus, they have a smaller vertical wave number. They may be identified with the geophysical Yanai waves (Yanai \& Maruyama 1966).

- Class $I V$ waves. They are purely prograde waves $(m<0)$ whose characteristics change little with $v_{\mathrm{M}}$, since their displacement in the $\theta$ direction is very small. Their dynamics are driven by the conservation of the specific vorticity combined with the stratification effects and by the Lorentz force; their eigenvalues are lower than those of both class $I$ and class III waves. Hence, their vertical wave number is smaller. In the hydrodynamical case, they may be identified with the geophysical Kelvin waves (Pedlosky 1998).

If we define a new index $s$ by

$s=l-m+1$ for $m>0$ or $s=l+m-1$ for $m \leq 0,(89)$

then Class $I V$ waves have $s=-1$, class III waves $s=0$, and class $I$ waves have $s=1,2,3, \ldots$ 

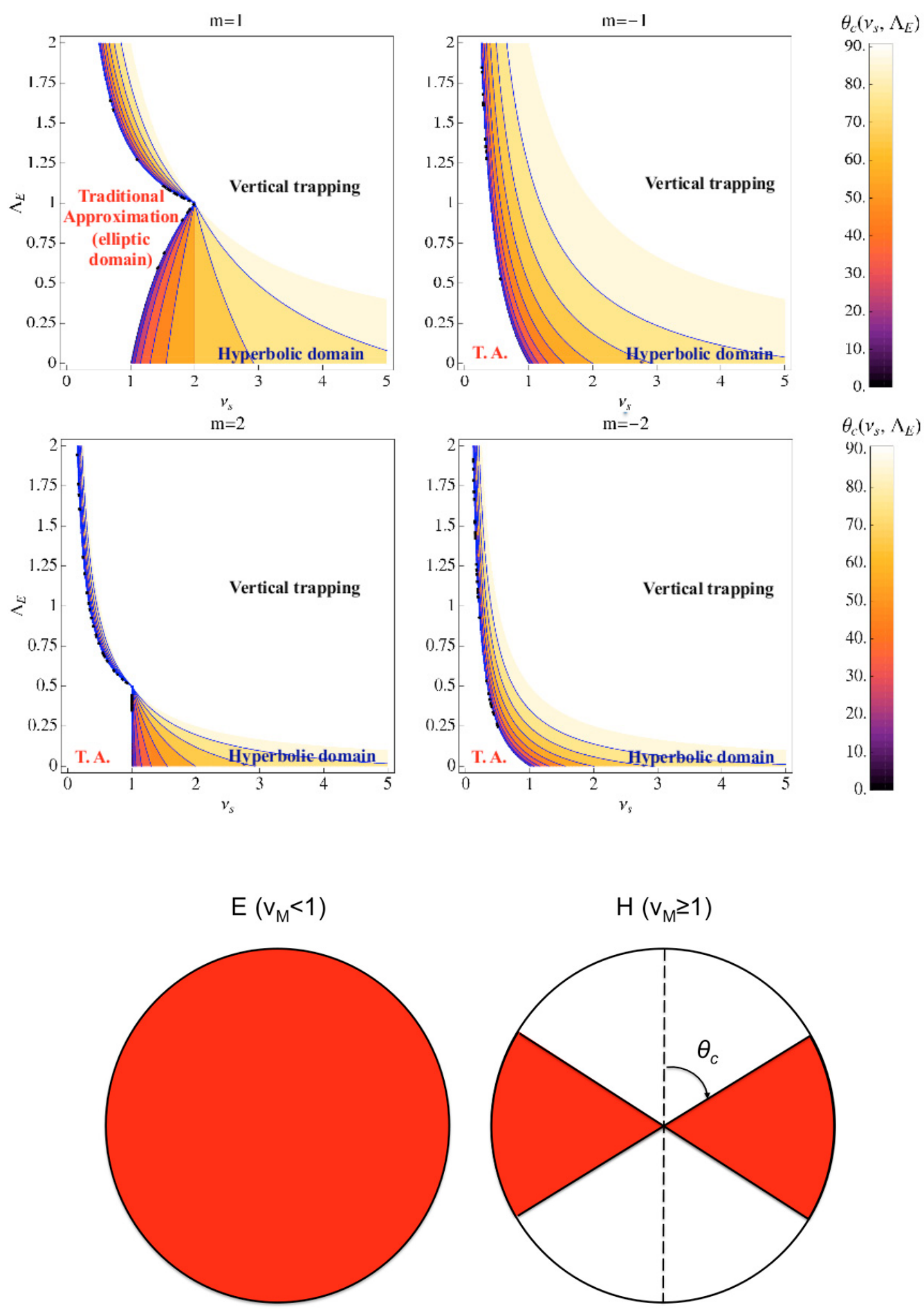

Fig. 7. Top: $\theta_{\mathrm{c}}$ as a function of $v_{\mathrm{s}}$ and $\Lambda_{\mathrm{E}}$ for non-axisymmetric retrograde $(m=1)$ and prograde $(m=-1)$ waves. As in Fig. (6), the large white top-right corner region corresponds to the $\left(v_{\mathrm{s}}, \Lambda_{\mathrm{E}}\right)$ domain where $\mathcal{A} \leq 0$ and where the waves are trapped in the vertical direction. In the white region at the left, the MHD TA applies (i.e. $\left|v_{\mathrm{M}}\right|<1$ and $\mathcal{A}>0$ ) and there is no critical latitude. Finally, the blue $\theta_{\mathrm{c}}$-iso lines correspond to the regime where the TA does not apply (i.e. $\left|v_{\mathrm{M}}\right| \geq 1$ and $\mathcal{A}>0$ ). Middle: same for $m=2$ and $m=-2$. Bottom: cartoon for the wave propagation region: traditional elliptic waves are regular for every latitude and live in the whole sphere $(\boldsymbol{E})$, while hyperbolic ones are trapped in the equatorial belt where $\theta \in\left[\theta_{\mathrm{c}}, \pi-\theta_{\mathrm{c}}\right](\boldsymbol{H})$.

\section{Conclusion and perspectives}

In this work, we examine of low-frequency internal wave behaviour in stellar radiation zones, which are stable highly stratified rotating magnetic regions. Then, waves dynamics are driven by the buoyancy force, the Coriolis acceleration, and the Lorentz force. Internal waves thus become magneto-gravitoinertial waves that are equivalent to the MAC waves (magneticArchimedean - Coriolis) which have been studied for the Earth's core. In this work in the stellar context, we studied the case 
of radiation zones with an axisymmetric purely toroidal magnetic field, where both the angular velocity $\left(\Omega_{\mathrm{S}}\right)$ and the Alfvén pulsation $\left(\omega_{\mathrm{A}}\right)$ are assumed to be uniform. This allowed to extract the main properties of the system. First, we derived dynamical equations, following Braginsky's work and using stellar notations. Then, the MHD TA, which can be used only in the strongly stratified case when $\left|v_{M}\right|<1$ and $\mathcal{A}>0$, has been introduced and discussed. This allows to simplify the equations system and to obtain in this case the wave-velocity field, the wave-magnetic field, and the total pressure fluctuation as a function of the usual Hough functions and associated special latitudinal and azimuthal functions used in the non-magnetic case for gravito-inertial waves in the super-inertial strongly stratified regime $\left(v_{\mathrm{s}}<1\right)$. Then, four classes of magneto-gravito-inertial waves are indentified, while the asymptotic behaviour of such low-frequency waves is discussed.

Once this study is achieved, two main works have to be engaged in the near future. The first one, is to examine the angular momentum transport by such waves in the "weak differential rotation case" (cf. Mathis et al. 2008), assuming a uniform Alfvén frequency to isolate the impact of an axisymmetric toroidal magnetic field as a function of its intensity (Paper II). Then, the more general case of general differential rotation and azimuthal magnetic field has to be studied, because it has been done in Mathis (2009) in the hydrodynamical case (Paper III). Moreover, the same methodology must be applied to axisymmetric poloidal magnetic fields. This will lead to a coherent picture of angular momentum wave-induced transport taking the impact of an axisymmetric fossil magnetic field into account.

Acknowledgements. Authors thank Drs. A.-S. Brun, S. Talon, S. Turck-Chièze, and J.-P. Zahn for fruitful discussions during this work, which was supported in part by the Programme National de Physique Stellaire (CNRS/INSU), the CNES/GOLF grant at the Service d'Astrophysique (CEA - Saclay), and the CNRS Physique théorique et ses interfaces programme.

\section{Appendix A: Magneto-gravito-inertial waves dispersion relation}

To establish the dispersion relation of magneto-gravito-inertial waves, we follow the method proposed in Kumar et al. (1999). As in our simplified model, we assume that the angular velocity is uniform $\left(\Omega=\Omega_{\mathrm{s}}\right)$, while the unperturbed magnetic field is here (and only here) assumed to also be uniform (i.e. $\boldsymbol{B}_{0}^{\mathrm{T}}=\overline{\boldsymbol{B}}$ ). We consider the short-wavelength limit, where spatial and temporal dependence of all perturbed quantities are taken as proportional to $\exp [\mathrm{i}(\boldsymbol{k} \cdot \boldsymbol{r}+\sigma t)]$. Then, the linearized momentum equation (Eq. (2)) is given by

$-\bar{\rho} \sigma^{2} \boldsymbol{\xi}+\bar{\rho} 2 \mathrm{i} \sigma \boldsymbol{\Omega} \times \boldsymbol{\xi}-\widetilde{\rho} \boldsymbol{g}+\mathrm{i} \boldsymbol{k} \widetilde{P}=\frac{\mathrm{i}(\boldsymbol{k} \times \boldsymbol{b})}{\mu} \times \overline{\boldsymbol{B}}$,

where $\boldsymbol{\Omega}=\Omega_{\mathrm{s}} \widehat{\boldsymbol{e}}_{z}$. Using the energy transport equation in the adiabatic limit (Eq. (30)), this becomes

$-\bar{\rho} \sigma^{2} \boldsymbol{\xi}+\bar{\rho} 2 \mathrm{i} \sigma \boldsymbol{\Omega} \times \boldsymbol{\xi}+\bar{\rho} N^{2} \xi_{r} \widehat{\boldsymbol{e}}_{r}+\mathrm{i} \boldsymbol{k} \widetilde{P}=\frac{\mathrm{i}(\boldsymbol{k} \times \boldsymbol{b})}{\mu} \times \overline{\boldsymbol{B}}$.

Substituting for $\boldsymbol{b}$ using the linearized induction equation (Eq. (1)) in the case of a perfectly conducting fluid and using the anelastic approximation that becomes in the short-wavelength $\boldsymbol{k} \cdot \boldsymbol{\xi}=0$, we obtain

$-\bar{\rho} \sigma^{2} \boldsymbol{\xi}+\bar{\rho} 2 \mathrm{i} \sigma \boldsymbol{\Omega} \times \boldsymbol{\xi}+\bar{\rho} N^{2} \xi_{r} \widehat{\boldsymbol{e}}_{r}+\mathrm{i} \boldsymbol{k} \widetilde{P}$

$+\frac{(\overline{\boldsymbol{B}} \cdot \boldsymbol{k})}{\mu}[(\overline{\boldsymbol{B}} \cdot \boldsymbol{k}) \boldsymbol{\xi}-(\overline{\boldsymbol{B}} \cdot \boldsymbol{\xi}) \boldsymbol{k}]=0$.
Taking the vector cross product of the previous equation with $\boldsymbol{k}$, we get

$\left[\sigma^{2}-(\widehat{\boldsymbol{B}} \cdot \boldsymbol{k})^{2} V_{A}^{2}\right](\boldsymbol{k} \times \boldsymbol{\xi})-N^{2} \xi_{r}\left(\boldsymbol{k} \times \widehat{\boldsymbol{e}}_{r}\right)+2 \mathrm{i} \sigma(\boldsymbol{\Omega} \cdot \boldsymbol{k}) \boldsymbol{\xi}=0,(\mathrm{~A} .4)$

where $V_{\mathrm{A}}^{2}=\overline{\boldsymbol{B}}^{2} /(\mu \bar{\rho})$ is the Alfvén velocity and $\widehat{\boldsymbol{B}}=\overline{\boldsymbol{B}} / \bar{B}$. Taking the cross product of the previous equation with $\boldsymbol{k}$ allows $\boldsymbol{k} \times \boldsymbol{\xi}$ as a function of $\boldsymbol{\xi}$. Substituting back in Eq. (A.4), we obtain

$\boldsymbol{\xi}\left\{-\frac{\left[\sigma^{2}-(\widehat{\boldsymbol{B}} \cdot \boldsymbol{k})^{2} V_{\mathrm{A}}^{2}\right]^{2} k^{2}}{2 \mathrm{i} \sigma(\boldsymbol{\Omega} \cdot \boldsymbol{k})}-2 \mathrm{i} \sigma(\boldsymbol{\Omega} \cdot \boldsymbol{k})\right\}$
$+\xi_{r}\left[N^{2}\left(\boldsymbol{k} \times \widehat{\boldsymbol{e}}_{r}\right)-\frac{N^{2}\left[\sigma^{2}-(\widehat{\boldsymbol{B}} \cdot \boldsymbol{k})^{2} V_{\mathrm{A}}^{2}\right]}{2 i \sigma(\boldsymbol{\Omega} \cdot \boldsymbol{k})} \boldsymbol{k} \times\left(\boldsymbol{k} \times \widehat{\boldsymbol{e}}_{r}\right)\right]=0$.

Taking the $r$-component of this equation gives the searched dispersion relation:

$$
\begin{aligned}
& {\left[\sigma^{2}-(\widehat{\boldsymbol{B}} \cdot \boldsymbol{k})^{2} V_{\mathrm{A}}^{2}\right]^{2}-(\boldsymbol{N} \times \widehat{\boldsymbol{k}})^{2}\left[\sigma^{2}-(\widehat{\boldsymbol{B}} \cdot \boldsymbol{k})^{2} V_{\mathrm{A}}^{2}\right]} \\
& -4 \sigma^{2}(\boldsymbol{\Omega} \cdot \widehat{\boldsymbol{k}})^{2}=0,
\end{aligned}
$$

where we have defined $\widehat{\boldsymbol{k}}=\boldsymbol{k} / \boldsymbol{k}$ and $\boldsymbol{N}=N \widehat{\boldsymbol{e}}_{r}$, which can be inverted:

$$
\begin{aligned}
\sigma^{2} & =(\widehat{\boldsymbol{B}} \cdot \boldsymbol{k})^{2} V_{\mathrm{A}}^{2}+\frac{1}{2}\left\{(\boldsymbol{N} \times \widehat{\boldsymbol{k}})^{2}+4(\boldsymbol{\Omega} \cdot \widehat{\boldsymbol{k}})^{2}\right. \\
& \left. \pm \sqrt{\left[(\boldsymbol{N} \times \widehat{\boldsymbol{k}})^{2}+4(\boldsymbol{\Omega} \cdot \widehat{\boldsymbol{k}})^{2}\right]^{2}+16(\widehat{\boldsymbol{B}} \cdot \boldsymbol{k})^{2} V_{\mathrm{A}}^{2}(\boldsymbol{\Omega} \cdot \widehat{\boldsymbol{k}})^{2}}\right\}
\end{aligned}
$$

Two branches can be isolated: the lower-frequency branch corresponds to the Alfvén waves modified by the Coriolis acceleration, and the higher-frequency one is linked to the magnetogravito-inertial waves.

\section{Appendix B: The magneto-gravity waves case}

In this short appendix, we simplify the general case treated here to unravel the magnetic field impact on gravity waves. We thus do not take the Coriolis acceleration into account (i.e. $\bar{\Omega}_{\mathrm{s}}=0$ ). Then, $v_{\mathrm{M}}$ becomes

$v_{\mathrm{M}}=-\frac{2 m \omega_{\mathrm{A}}^{2}}{\sigma^{2}-m^{2} \omega_{\mathrm{A}}^{2}}$,

and the waves' horizontal structure is described by associated Hough functions. Moreover, the MHD TA can be applied as long as $\left|v_{\mathrm{M}}\right|<1$, which corresponds to

$\sigma^{2}>\omega_{\mathrm{A}}^{2}\left(m^{2}+2|m|\right)$

for propagative waves for which $\mathcal{A}=\sigma^{2}-m^{2} \omega_{\mathrm{A}}^{2}>0$.

\section{References}

Acheson, D. J. 1978, R. Soc. London Phil. Trans. Ser. A, 289, 459 Antia, H. M., Chitre, S. M., \& Thompson, M. J. 2003, A\&A, 399, 329 Barnes, G., MacGregor, K. B., \& Charbonneau, P. 1998, ApJ, 498, L169 Belkacem, K., Samadi, R., Goupil, M. J., et al. 2009, A\&A, 494, 191 Bildsten, L., Ushomirsky, G., \& Cutler, C. 1996, ApJ, 460, 827 
Braginsky, S. I. 1967, Geomagnetism and Aeronomy, 7, 851

Braginsky, S. I., \& Roberts, P. H. 1975, Proc. Royal Soc. London A, 347, 125 Braithwaite, J. 2009, MNRAS, 397, 763

Braithwaite, J., \& Spruit, H. C. 2004, Nature, 431, 819

Browning, M. K., Miesch, M. S., Brun, A. S., \& Toomre, J. 2006, ApJ, 648, L157

Brun, A.-S., \& Zahn, J.-P. 2006, A\&A, 457, 665

Charbonnel, C., \& Talon, S. 2005, Science, 309, 2189

Cowling, T. G. 1941, MNRAS, 101, 367

Denissenkov, P. A., Pinsonneault, M., \& MacGregor, K. B. 2008, ApJ, 684, 757

Dintrans, B., \& Rieutord, M. 2000, A\&A, 354, 86

Dintrans, B., Rieutord, M., \& Valdettaro, L. 1999, J. Fluid Mech., 398, 271

Dintrans, B., Brandenburg, A., Nordlund, Å, \& Stein, R. F. 2005, A\&A, 438, 365

Duez, V., \& Mathis, S. 2010, A\&A, 517, A58

Eckart, C. 1960, Hydrodynamics of Oceans and Atmospheres (Oxford: Pergamon Press)

Friedlander, S. 1987a, Geophys. J. Royal Astron. Soc., 89, 637

Friedlander, S. 1987b, Geophys. Astrophys. Fluid Dyn., 39, 315

Friedlander, S. 1989, Geophys. Astrophys. Fluid Dyn., 48, 53

Friedlander, S., \& Siegmann, W. L. 1982, Geophys. Astrophys. Fluid Dyn., 19, 267

Fruman, M. D. 2009, J. Atmos. Sci., 66, 2937

Garaud, P., \& Guervilly, C. 2009, ApJ, 695, 799

Gerkema, T., \& Shrira, V. I. 2005, J. Fluid Mech., 529, 195

Gerkema, T., Zimmerman, J. T. F., Mass, L. R. M., \& Van Haren, H. 2008, Rev. Geophys., 46, CiteID RG2004

Gough, D. O., \& McIntyre, M. E. 1998, Nature, 394, 755

Heng, K., \& Spitkovsky, A. 2009, ApJ, 703, 1819

Hough, S. S. 1898, Philos. Trans. Royal Soc. London A, 191, 139

Kiraga, M., Jahn, K.; Stepien, K., \& Zahn, J.-P. 2003, Acta Astron., 53, 321

Kiraga, M., Stepien, K., \& Jahn, K. 2005, Acta Astron., 55, 205

Kumar, P., Talon, S., \& Zahn, J.-P. 1999, ApJ, 520, 859

Landau, L. D., \& Lifchitz, E. M. 1966, Theoretical Physics: Quantum Mechanics (Moscow: Mir)

Laplace, P.-S. 1799, Mécanique céleste, Bureau des Longitudes, Paris

Lee, U. 2005, MNRAS, 357, 97

Lee, U. 2007, MNRAS, 374, 1015

Lee, U. 2010, MNRAS, 405, 1444

Lee, U., \& Saio, H. 1987, MNRAS, 225, 643

Lee, U., \& Saio, H. 1989, MNRAS, 237, 875
Lee, U., \& Baraffe, I. 1995, A\&A, 301, 419

Lee, U., \& Saio, H. 1997, ApJ, 491, 839

Lindzen, R. S., \& Chapman, S. 1969, Space Sci. Rev., 10, 3

Longuet-Higgins, M. S. 1968, Philos. Trans. Royal Soc. of London A, 262, 511

Maas, L., \& Harlander, U. 2007, J. Fluid Mech., 570, 47

Markey, P., \& Tayler, R. J. 1973, MNRAS, 163, 77

Markey, P., \& Tayler, R. J. 1974, MNRAS, 168, 505

Mathis, S. 2009, A\&A, 506, 811

Mathis, S., Talon, S., Pantillon, F.-P., \& Zahn, J.-P. 2008, Sol. Phys., 251, 101

Meynet, G., \& Maeder, A. 2000, A\&A, 361, 101

Mestel, L., Tayler, R. J., \& Moss, D. L. 1988, MNRAS, 231, 873

Pantillon, F. P., Talon, S., \& Charbonnel, C. 2007, A\&A, 474, 155

Pedlosky, J. 1998, Geophysical fluid dynamics, 2nd edn. (Springer)

Press, W. 1981, ApJ, 245, 286

Provost, J., Berthomieu, G., \& Rocca, G. 1981, A\&A, 94, 126

Rogers, T. M., \& Glatzmaier, G. A. 2005, MNRAS, 364, 1135

Rogers, T. M., \& Glatzmaier, G. A. 2006, ApJ, 653, 756

Rogers, T. M., \& MacGregor, K. B. 2010, MNRAS, 401, 191

Schatzman, E. 1993a, A\&A, 271, L29

Schatzman, E. 1993b, A\&A, 279, 431

Schecter, D. A., Boyd, J. F., \& Gilman, P. A. 2001, ApJ, 551, L185

Stewartson, K., \& Richard, J. 1969, J. Fluid Mech., 35, 759

Stewartson, K., \& Walton, I. C. 1976, Proc. Royal Soc. London A, 349, 141

Talon, S. 2008, EAS PS, 32, 81

Talon, S., \& Charbonnel, C. 2005, A\&A, 440, 981

Talon, S., Kumar, P., \& Zahn, J.-P. 2002, ApJ, 574, L175

Tayler, R. J. 1973, MNRAS, 161, 365

Tayler, R. J. 1980, MNRAS, 191, 151

Townsend, R. H. D. 2003, MNRAS, 340, 1020

Unno, W., Osaki, Y., Ando, H., Saio, H., \& Shibahashi, H. 1989, Non-radial oscillations of stars, second edition (Tokyo: University of Tokyo Press)

Wright, G. A. E. 1973, MNRAS, 162, 339

Yanai, M., \& Maruyama, T. 1966, J. Meteorol. Soc. Japan, 44, 291

Zahn, J.-P. 1970, A\&A, 4, 452

Zahn, J.-P. 1975, A\&A, 41, 329

Zahn, J.-P. 1992, A\&A, 265, 115

Zahn, J.-P., Talon, S., \& Matias, J. 1997, A\&A, 322, 320

Zaqarashvili, T. V., Olivier, R., Ballester, J. L., \& Shergelashvili, B. M. 2007, A\&A, 470, 815

Zaqarashvili, T. V., Olivier, R., \& Ballester, J. L. 2009, ApJ, 691, L41 